\title{
Errata
}

\section{Erratum: Possible Advantages of a Robust Evaluation of Comparisons}

\author{
Jörg Müller \\ Bureau International des Poids et Mesures, \\ F-92312 Sevres, France
}

[J. Res. Natl. Inst. Stand. Technol. Volume 105, Number 4, July-August 2000, p. 551]

On p. 554, the sixth line down in the right-hand column should be as follows:

If $t$ is located between $y_{-1}$ and $y_{1}: Q=y_{1}-y_{-1}=Q_{0}$. 